\title{
The Mathematical Modeling and Proof of the Goldbach Conjecture
}

\author{
Yu Wang \\ College of Information Science \& Technology, Chengdu University of Technology, China
}

\begin{abstract}
The Goldbach conjecture declares that any even number $2 m=2 n+2>4$ can be expressed as the sum of two prime numbers. The mathematical modeling of the conjecture is: any even number $2 m=2 n+2$ greater than 4 can be expressed as $2 n+2=a+b, 2 \leq a \leq n+1, n+1 \leq b \leq 2 n$. With the modeling, let $c$ be a composite number in $2 \sim 2 n$, a mapping number is $2 \mathrm{~m}-\mathrm{c}$ or $2 \mathrm{n}+2-\mathrm{c}$. A complete composite pair is a pair (c, $2 \mathrm{~m}-\mathrm{c})$ that both $c$ and $2 \mathrm{~m}-\mathrm{c}$ are composite numbers. The composite numbers one-to-one correspond to the mapping numbers. Using an induction to absurdity, suppose the Goldbach conjecture is wrong, so that $2 n+2$ cannot be expressed as the sum of two primes. With the mathematical modeling for the even number $2 n+2$, numbers $2 \sim 2 n$ are all composite numbers or mapping numbers. A false inequation (C) can be obtained when $n \geq 128$. This means that the supposition does not stand when $n \geq 128$. Meanwhile, the Goldbach conjecture can be easily verified for the even numbers in 6 256. Hence, the Goldbach conjecture is proved.
\end{abstract}

Keywords-Goldbach conjecture; composite number pair; mapping number; induction to absurdity

\section{NOTATION}

$e \quad$ An even number greater than $4 ; \mathrm{e}=2 \mathrm{~m}=2 \mathrm{n}+2$.

$(a, b)$ A decomposition pair. a and $b$ are natural numbers, $e=a+b$.

$a \sim b \quad$ The natural number range from $a$ to $b$. A natural number $x$ in the range satis fies $a \leq x \leq b$.

$p \quad$ A prime number.

[x] The largest integer less than or equal to $x$.

$\mathrm{n}$ ! The factorial computation, meaning $\mathrm{n} \times(\mathrm{n}-1) \times \ldots 2 \times 1$.

$C_{n}^{k} \quad C_{n}^{k}=\frac{n !}{k !(n-k) !}$

$C_{2 n}^{n} \quad C_{2 n}^{n}=\frac{(2 n) \times(2 n-1) \times \ldots(n+1)}{1 \times 2 \times 3 \times \ldots n}=\frac{(2 n) \times(2 n-1) \times \ldots(n+1)}{2 \times 3 \times \ldots n}$

$\prod_{p \leq n} p$ The product of all the prime numbers less than or equal to $n$.

$\mathrm{a} b \quad$ Divide exactly. Integer $b$ is a multiple of integer $a$.

$\mathrm{a} \nmid \mathrm{b} \quad$ Not divide exactly. Integer $b$ is not a multiple of integer $a$.

$\mathrm{i}_{\mathrm{p}} \quad$ The minimum mapping number in 2 n corresponding to the maximum composite number in $(n+1) \sim 2 n$ produced by prime $p, 2 \leq \mathrm{i}_{\mathrm{p}} \leq \mathrm{p}+1$.

$\mathrm{L} \quad \mathrm{L}=\left[\frac{2 n}{p}\right]$

composite numbers in $(n+1) \sim 2 n$

composite numbers in $1 \sim n$ it is
For example, when $n=10$,

\section{INTRODUCTION}

Three major mathematical conjectures include Fermat's Conjecture, Four Color Conjecture and Goldbach Conjecture. The former two conjectures have been proved as Fermat's Last Theorem and Four color theorem. The Goldbach conjecture (referred as the conjecture) is a bright pearl in the field of mathematics and is regarded as unproved till now. It was raised in 1742 in a letter from Goldbach to Euler. In 1900, D. Hilbert proposed the Goldbach conjecture as one of the twenty-three unsolved problems. The Goldbach's conjecture is worth studying. Solving the conjecture promotes a breakthrough in related fields, especially in the field of number theory, leading to a series of related progress[1][2].

A common referred expression for the Goldbach conjecture is: any even number greater than or equal to 6 (or greater than 4 ) can be expressed as the sum of two odd prime numbers. The conjecture is also called as " $1+1$ " or " $1+1=2$ ". The problem is very difficult. In many journals, periodicals and Internet resources, enthusiasts have shared their "proofs" for the Goldbach's conjecture. As far as I know, the problem has not been solved. So far, the best work about the conjecture is the proof of " $1+2$ " by Chen Jinrun.

\section{RELATED STUDIES ON THE GOLDBACH CONJECTURE}

Geniuses have poured great enthusiasms and made great efforts on the proof of the Goldbach conjecture. As far as I know, in most existing literatures, researchers verify or prove irrigorously the Goldbach conjecture, or research on similar propositions to the Goldbach's conjecture. This means that the proof of the Goldbach conjecture has not been successful. The best contribution on Goldbach's conjecture is made by China's Chen Jingrun. Chen Jingrun proves that every sufficiently large even number can be written as the sum of either two primes, or a prime and a semiprime (the product of two primes). His contribution is called as " $1+2$ ". There are also other researches [3]. However, these researches do not prove the conjecture itself.

The Goldbach conjecture brings other topics relevant with the Goldbach conjecture. Reference [4] discusses the sum of four prime numbers. Reference [5] verifies "ternary Goldbach conjecture". Reference [6] further improves Hua Luogeng's work. Some works are not the proofs of the Goldbach conjecture[7]. The study of the conjecture promotes progresses in the field of number theory. For example, prime distribution rule [8] is a harvest in the study of the conjecture. 


\section{RELATED PROPERTIES OF NUMBER THEORY}

Definition $1 \mathrm{~A}$ prime number is a natural number that can only be divided exactly by 1 and itself. A composite number can be divided exactly by 1 , itself and other natural numbers.

Definition 2 Let $\mathrm{p}$ be a prime number, $\mathrm{k}$ be a natural number and $\mathrm{k}>1$, we call $\mathrm{kp}$ or $\mathrm{k} \times \mathrm{p}$ as $\mathrm{p}$ 's composite number, or the composite number $\mathrm{kp}$ is produced by $\mathrm{p}$. When $\mathrm{k} \geq 1$, $\mathrm{kp}$ is p's multiple, or $\mathrm{kp}$ is a multiple number produced by $\mathrm{p}$.

The multiples produced by the prime $p$ are $p, 2 p, 3 p, 4 p \ldots$ The composite numbers produced by $\mathrm{p}$ are $2 \mathrm{p}, 3 \mathrm{p}, 4 \mathrm{p} \ldots$

Let $g$ be a composite number greater than 4 , as $g=\sqrt{g} \times \sqrt{g}$, its smallest prime factor is less than or equal to $\sqrt{g}$; i.e., a composite number g must have at least one prime factor that is less than or equal to $\sqrt{g}$. Because the smallest prime number is 2 , a composite number $g$ is divisible exactly by a prime number that is less than or equal to $\frac{2}{2}$.

When $\mathrm{n}=10$, the number of prime numbers is not greater $\mathrm{n}$ than $\overline{2}-1$. When $n \geq 10$, any even number is not prime. Therefore, the number of prime numbers not greater than $\mathbf{n} \leq \frac{\mathbf{n}}{2}-1$, $\mathrm{n} \geq 10$.

Definition 3 Let $x$ be a real number, [x] is equal to the largest integer less than or equal to $x$.

Property 1 Let $x \geq 0$, then $[x] \leq x, 2[x]+1 \geq[2 x] \geq 2[x]$.

Proof: When $x \geq 0$, let $x=a+b, a=[x], 0 \leq b<1$; then $[x] \leq x$, $2 \mathrm{x}=2 \mathrm{a}+2 \mathrm{~b}$, so $[2 \mathrm{x}]=[2 \mathrm{a}+2 \mathrm{~b}]=\left\{\begin{array}{c}2[\mathrm{x}]+1,1>\mathrm{b} \geq 0.5 \\ 2[\mathrm{x}], 0 \leq \mathrm{b}<0.5\end{array}\right.$.

Hence $2[\mathrm{x}]+1 \geq[2 \mathrm{x}] \geq 2[\mathrm{x}]$.

An equivalent expression of property 1 is $0 \leq[2 \mathrm{x}]-2[\mathrm{x}]$ $\leq 1$.

Property 2 Let a, b, i be integers,

(i) $a>b>0$, i $>0$, then $\frac{a+i}{b+i}<\frac{a+i-1}{b+i-1} \leq \frac{a}{b}$;

(ii) $a>i \geq 2, a>b, b=0$, then $\frac{a+i}{b+i}<a$.

Proof: Let a, b, i be integers

(i) When $a>b>0$, i $>0$ then $\frac{a+i}{b+i}-\frac{a}{b}=\frac{i(b-a)}{b(b+i)}<0$, so $\frac{a+i}{b+i}<\frac{a}{b}$.

From $“ \frac{a+1}{b+i}<\frac{a}{b}$, we get $\frac{a+1}{b+i}=\frac{a+i-1+1}{b+i-1+1}<\frac{a+i-1}{b+i-1}$;

for $i-1 \geq 0, \frac{a+i-1}{b+i-1} \leq \frac{a}{b}$.

Hence we get $\frac{a+1}{b+i}<\frac{a+i-1}{b+i-1} \leq \frac{a}{b}$.

(ii) When $\quad a>i \geq 2, \quad a>b, \quad b=0$, then $\frac{a+i}{b+i}-a=\frac{a+i}{i}-a=\frac{(1-i) a+i}{i} \leq \frac{(1-2) a+i}{i}<0$. Therefore, $\frac{a+i}{b+i}<a$.

Theorem 1 Let $n$ be a positive integer, then $C_{2 n}^{n} \geq \frac{4^{n}}{2 n}$.

Proof: Let $k$ be a positive integer, $C_{2 n}^{n}>C_{2 n}^{k}$ when $k \neq n$.
Meanwhile, $C_{2 n}^{n} \geq 2=C_{2 n}^{0}+C_{2 n}^{2 n}$.

Hence $2 n C_{2 n}^{n} \geq \sum_{i=0}^{2 n} C_{2 n}^{i}=2^{2 n}=4^{n}$.

Therefore, $C_{2 n}^{n} \geq \frac{4^{n}}{2 n}$.

Corollary 1.1 Let $n$ be a positive integer, then $C_{2 n+1}^{n}=C_{2 n+1}^{n+1} \geq \frac{2 \times 4^{n}}{2 n+1}$

Proof: Let $k$ be a positive integer, $C_{2 n+1}^{n}>C_{2 n+1}^{k}$ when $\mathrm{k} \neq \mathrm{n}$ and $\mathrm{k} \neq \mathrm{n}+1$.

Meanwhile, $C_{2 n+1}^{n} \geq 2=C_{2 n+1}^{0}+C_{2 n+1}^{2 n+1}$.

Hence, $(2 n+1) C_{2 n+1}^{n} \geq \sum_{i=0}^{2 n+1} C_{2 n+1}^{1}=2^{2 n+1}=2 \times 4^{n}$. Therefore, $C_{2 n+1}^{n} \geq \frac{2 \times 4^{n}}{2 n+1}$.

Corollary $1.2 \mathrm{n} \geq 2$. When $n$ is an even number, $C_{n}^{\frac{n}{2}} \geq \frac{4^{\frac{n}{2}}}{n}$; when $n$ is an odd number, $C_{n}^{\frac{n-1}{2}}=C_{n}^{\frac{n+1}{2}} \geq=\frac{4 \frac{n}{2}}{n}$.

Proof: $n$ is a positive number, $n \geq 2$. From theorem 1, when $\mathrm{n}$ is a positive even number, $C_{n}^{\frac{n}{2}} \geq \frac{4 \frac{n}{2}}{n}$.

From Corollary 1.1, when $\mathrm{n}$ is a positive odd number, $C_{n}^{\frac{n-1}{2}}=C_{n}^{\frac{n+1}{2}} \geq 2 \times \frac{4 \frac{n-1}{2}}{n}=\frac{4 \frac{n}{2}}{n}$.

Notice that the right side is $\frac{4 \frac{n}{2}}{n}$ whether $n$ is an even number or an odd number.

Lemma 1 Let $p$ be a prime number, $k$ be a natural number, then $\prod_{k+1<p \leq 2 k+1} p<4^{k}$.

Proof: Let $\mathrm{p}$ be a prime number, $\mathrm{k}$ be a natural number. All the prime numbers satisfying $\mathrm{k}+1<\mathrm{p} \leq 2 \mathrm{k}+1$ are in $(2 \mathrm{k}+1)$ !, not in $(\mathrm{k}+1)$ ! or $\mathrm{k}$ !. Hence $\prod_{k+1<p \leq 2 k+1} p$ is factor of $C_{2 k+1}^{k+1}$. Thus $\prod_{k+1<p \leq 2 k+1} p_{\mid} C_{2 k+1}^{k+1}$ Hence $\prod_{k+1<p \leq 2 k+1} p_{\leq} C_{2 k+1}^{k+1}$. And $2^{C_{2 k+1}^{k+1}}=C_{2 k+1}^{k}+C_{2 k+1}^{k+1}<\sum_{i=0}^{2 k+1} C_{2 k+1}^{i}=2^{2 k+1}=2 \times 4^{k}$, so $C_{2 k+1}^{k+1} 4^{k}$.

Therefore, $\prod_{k+1<p \leq 2 k+1} p_{\leq} C_{2 k+1}^{k+1} 4^{k}$. Proved.

Theorem 2 Let $n$ be a positive integer, $p$ be a prime number, then $\prod_{p \leq n} p<4^{n}$.

Proof: Using mathematical induction. When $n=2,2<16$, it is satisfied.

Suppose the theorem is satisfied for all the positive integer numbers less than $\mathrm{n}$.

If $\mathrm{n}>2$ and $\mathrm{n}$ is an even number, $\prod_{p \leq n} p=\prod_{p \leq n-1} p$.

If $n$ is an odd number, let $n=2 k+1$. From Lemma 1 and the inductive supposition, we get

$\prod_{p \leq n} p=\prod_{p \leq 2 k+1} p=\prod_{p \leq k+1} p \times \prod_{k+1<p \leq 2 k+1} p<4^{k+1} \times 4^{k}=4^{2 k+1}=4^{n}$

Proved. 


\section{MATHEMATICAL MODELINGS OF THE GOLDBACH CONJECTURE}

\section{A. Primitive Mathematical Modelings of the Goldbach Conjecture}

Any even number $e$ not less than 6 can be expressed as the sum of two natural numbers; i.e., $\mathrm{e}=\mathrm{a}+\mathrm{b}, 0<\mathrm{a}, \mathrm{b}<\mathrm{e}$. The $a$ is located in the left column, $b$ in the right column, and we name (a, b) as a decomposition pair. For short, a is in the left, b is in the right. The corresponding decomposition of $a$ and $b$ is listed in the left and right columns of table 1.

TABLE I. THE ENTIRE LEFT-RIGHT DECOMPOSIT IONS OF AN EVEN NUMBER $E, E \geq 6, E=A+B$

\begin{tabular}{|c|c|}
\hline $\begin{array}{c}\text { left column } \\
\mathbf{a}\end{array}$ & $\begin{array}{c}\text { right column } \\
\mathbf{b}\end{array}$ \\
\hline 1 & $\mathrm{e}-1$ \\
\hline 2 & $\mathrm{e}-2$ \\
\hline 3 & $\mathrm{e}-3$ \\
\hline$\ldots$ & $\ldots$ \\
\hline $\mathrm{e}-3$ & 3 \\
\hline $\mathrm{e}-2$ & 2 \\
\hline $\mathrm{e}-1$ & 1 \\
\hline
\end{tabular}

In table $1, \mathrm{e}=\mathrm{a}+\mathrm{b}, 0<\mathrm{a}, \mathrm{b}<\mathrm{e}, e$ is an even, $a$ and $b$ are natural numbers. It is natural that we list the numbers from small to large in the left column, and from large to small in the right column accordingly. Each decomposition pair (a, b) has the relation $\mathrm{a}+\mathrm{b}=\mathrm{e}$. Any number in the left is one-to-one corresponding to another number in the right when $e$ is expressed as two addend numbers $a$ and $b$ according to the Goldbach conjecture.

Definition 4 (a, b) is an even pair when $a$ and $b$ are even numbers; (a, b) is an odd pair when $a$ and $b$ are odd numbers; (a,b) is a prime pair when $a$ and $b$ are prime numbers; $(\mathrm{a}, \mathrm{b})$ is a composite pair when at least one number of $a$ and $b$ is a composite number.

There are duplicate decomposition pairs in table 1; i.e., there exist decomposition pairs which have the same numbers only with a different order. For example, $(3,5)$ and $(5,3)$ have the same numbers and with only different orders. Table 2 lists out the left-right decomposition of the Goldbach conjecture without duplicate pairs.

TABLE II. THE LEFT-RIGHT DECOMPOSITION OF E. E IS AN EVEN NUMBER, $\mathrm{E} \geq 6, \mathrm{E}=\mathrm{A}+\mathrm{B}$

\begin{tabular}{|c|c|}
\hline $\begin{array}{c}\text { left column } \\
\boldsymbol{a}\end{array}$ & $\begin{array}{c}\text { right column } \\
\boldsymbol{b}\end{array}$ \\
\hline 1 & $\mathrm{e}-1$ \\
\hline 2 & $\mathrm{e}-2$ \\
\hline 3 & $\mathrm{e}-3$ \\
\hline$\ldots$ & $\ldots$ \\
\hline $\mathrm{e} / 2-2$ & $\mathrm{e} / 2+2$ \\
\hline $\mathrm{e} / 2-1$ & $\mathrm{e} / 2+1$ \\
\hline $\mathrm{e} / 2$ & $\mathrm{e} / 2$ \\
\hline
\end{tabular}

According to the addition exchange law, table 2 can represent all the decomposition pairs in table 1 . In order to express and observe intuitively the inherent properties of the Goldbach conjecture, we replace the even number $e$ with $2 \mathrm{~m}$, and get table 3. $m$ is the shared number in the left column and right column. $2 \mathrm{~m}=\mathrm{a}+\mathrm{b}, 1 \leq \mathrm{a} \leq \mathrm{m}, \mathrm{m} \leq \mathrm{b} \leq 2 \mathrm{~m}-1$.

TABLE III. THE LEFT-RIGHT DECOMPOSITION OF THE GOLDBACH CONJECT URE. $1<\mathrm{A} \leq \mathrm{M}, \mathrm{M}<\mathrm{B} \leq 2 \mathrm{M}-1,2 \mathrm{M}>6,2 \mathrm{M}=\mathrm{A}+\mathrm{B}$

\begin{tabular}{|c|c|}
\hline $\begin{array}{c}\text { left column } \\
\mathbf{a}\end{array}$ & $\begin{array}{c}\text { right column } \\
\mathbf{b}\end{array}$ \\
\hline 1 & $2 \mathrm{~m}-1$ \\
\hline 2 & $2 \mathrm{~m}-2$ \\
\hline 3 & $2 \mathrm{~m}-3$ \\
\hline$\ldots$ & $\ldots$ \\
\hline $\mathrm{m}-2$ & $\mathrm{~m}+2$ \\
\hline $\mathrm{m}-1$ & $\mathrm{~m}+1$ \\
\hline $\mathrm{m}$ & $\mathrm{m}$ \\
\hline
\end{tabular}

The left column of table 3 includes all the natural numbers from 1 to $m$, the right column includes all the natural numbers from $2 \mathrm{~m}-1$ to $m$ correspondingly. According to the addition exchange law $\mathrm{a}+\mathrm{b}=\mathrm{b}+\mathrm{a}$, The decomposition pairs in table 3 represent all the decomposition pairs in table 1.

Lemma 2 Equivalent proposition No.1 of the Goldbach conjecture: There is at least one prime pair in table 3; i.e., at least one row in table 3 contains only prime numbers.

Proof: When the Goldbach conjecture is right, there are two prime numbers $a$ and $b$ whose sum is $2 \mathrm{~m}$. Suppose $a$ is less than or equal to $b$, then $1 \leq \mathrm{a} \leq \mathrm{m}, \mathrm{m} \leq \mathrm{b} \leq 2 \mathrm{~m}-1$, so the prime pair (a, b) must exist in table 3 .

When there is one prime pair $(a, b)$ in table 3 , then $a+b=2 m$, the Goldbach conjecture is right.

Therefore, "There is at least one prime pair in table 3" is both necessary condition and sufficient condition of the Goldbach conjecture. Proved.

Lemma 2 shows that the Goldbach conjecture implies that there must be a prime pair for an even number $2 \mathrm{~m}$ in table 3 .

\section{B. Formal Left-right Decomposition of the Conjecture}

Lemma 3 Equivalent proposition No.2 of the Goldbach conjecture: except $(1,2 \mathrm{~m}-1)$, at least one prime pair $(\mathrm{a}, \mathrm{b})$ exists among the other decomposition pairs in table 3.

Proof: Number 1 is neither a prime number, nor a composite number. The only difference between lemma 3 and lemma 2 is that lemma 3 excludes one decomposition pair (1, $2 \mathrm{~m}-1$ ), so the equivalent proposition No.2 is also right.

According to Lemma 3, we only need to study the decomposition pairs when a $>1$. Let $2 n=2 m-2$, we can get table 4 that excludes the decomposition pair $(1,2 m-1) . m$ is in the left, and is also in the right; $m$ is not in $1 \sim n$, but in $(n+1) \sim 2 n$. 
TABLE IV. THE LEFT-RIGHT DECOMPOSITION OF THE GOLDBACH CONJECT URE. $2 \leq \mathrm{A} \leq \mathrm{N}+1, \mathrm{~N}+1 \leq \mathrm{B} \leq 2 \mathrm{~N}, \mathrm{~A}+\mathrm{B}=2 \mathrm{~N}+2, \mathrm{M}=\mathrm{N}+1,2 \mathrm{M} \geq 6$

\begin{tabular}{|c|c|}
\hline $\begin{array}{c}\text { left column } \\
\boldsymbol{a}\end{array}$ & $\begin{array}{c}\text { right column } \\
\boldsymbol{b}\end{array}$ \\
\hline 2 & $2 \mathrm{n}$ \\
\hline 3 & $2 \mathrm{n}-1$ \\
\hline$\cdots$ & $\cdots$ \\
\hline $\mathrm{m}-2$ & $\mathrm{~m}+2$ \\
\hline $\mathrm{m}-1$ & $\mathrm{~m}+1$ \\
\hline $\mathrm{m}$ & $\mathrm{m}$ \\
\hline
\end{tabular}

According to Lemma 3, we get:

Lemma 4 Equivalent proposition No.3 of the Goldbach conjecture: there is at least one prime pair $(\mathrm{a}, \mathrm{b})$ in table 4.

Lemma 4 and Lemma 3 have the same meaning. Lemma 4 uses $2 n$ to substitute $2 \mathrm{~m}-2$.

The numbers in the left and right column in table 4 includes all the composite and prime numbers from 2 to $2 \mathrm{n}$. Any composite number in the left or right column in table 4 can be expressed as pk (short for $\mathrm{p} \times \mathrm{k}$ ), where $\mathrm{p}$ is a prime number not greater than $n$, and $k$ is a natural number between 2 and $n$. The multiples of prime $p$ are $p, 2 p, 3 p, \ldots k p, \ldots$ The first number $p$ is the only prime number; the othernumbers are composite.

\section{Mapping Numbers}

Let $p$ be a prime number not greater than $n$. We focus on the corresponding numbers to p's composite numbers in table 4 , where $\mathrm{a}+\mathrm{b}=2 \mathrm{n}+2$. When $b$ is p's maximu $\mathrm{m}$ composite number in the right, and let $\mathrm{i}_{\mathrm{p}}$ be its corresponding number in the left, then $2 \leq i_{p} \leq p+1$. p's maximal composite number is $\left\langle\frac{2 n}{p}\right\rangle$, hence $<\frac{2 n}{p}>+i_{p}=2 n+2$. Any corresponding number in the left to a composite number in the right has the form of $\mathrm{kp}^{+\mathrm{i}_{\mathrm{p}}}$, $\mathrm{k}$ is a nonnegative integer. Any corresponding number in the right to p's composite number in the left also has the form of $\mathrm{kp}+\mathrm{i}_{\mathrm{p}}$.

Suppose $a$ is a composite number in $2 \sim 2 n$, then $b=2 n+2-a$ is a's mapping number. Suppose $a$ is p's composite number in $2 \sim 2 n$, then $b=2 n+2-a$ is also called as p's mapping number. p's mapping numbers have the form of $\mathrm{kp}+\mathrm{i}_{\mathrm{p}}$ corresponding to prime $\mathrm{p}$. The mapping number $\mathrm{kp}+\mathrm{i}_{\mathrm{p}}$ is also called as a mapping number of prime number $\mathrm{p}$. For example, when $2 \mathrm{n}=34, \mathrm{p}=5$, the mapping number in the left is 6 correspoing to 30 in the right; the mapping number in the right is 21 correspoing to 15 in the left; $i_{p}=6$. Notice that when $p \mid m, m$ is a special number. The p's composite number and mapping number is $m$ itself.

$i_{p}$ is the minimum mapping number in the left corresponding to the maximum composite number in the right produced by $\mathbf{p}, \mathbf{2} \leq \mathbf{i}_{\mathbf{p}} \leq \mathbf{p}+\mathbf{1}$. $2 n+2-2 p$ is the maximum mapping number in the right corresponding to the minimum composite produced by $\mathrm{p}$ in the left, $\mathrm{m} \geq 2 \mathrm{p}$.

A composite pair $(a, b)$ is a decomposition pair in which at least one addend of $a$ and $b$ is a composite. This means: (i) The number a in the left is either a composite number, or a mapping number corresponding to another composite number in the right; (ii) The number $\mathrm{b}$ in the right is either a composite number, or a mapping number corresponding to another composite number in the left.
A complete composite pair (a,b) is a composite pair when both a and b are composite numbers; an incomple te composite pair (a,b) is a composite pair when only one member of a and b is composite. A composite pair is either a complete composite pair or an incomplete composite pair.

The Goldbach conjecture means that there exists at least one prime pair (a, b) to an even number $2 n+2$, so we get:

Theorem 3 A sufficient condition of the Goldbach conjecture: there exists at least one number in $2 \sim 2 n$ that is neither a composite number nor a mapping number.

Proof: Suppose there exist a natural number $\mathrm{g}, 2 \leq \mathrm{g} \leq 2 \mathrm{n}$, and $\mathrm{g}$ is neither a composite number nor a mapping number. Then the decomposition ( $\mathrm{g}, 2 \mathrm{n}-\mathrm{g}$ ) is not a composite pair. It is a prime pair. The Goldbach conjecture is right then. Proved.

\section{The Numerator and denominator of $\mathbf{C}_{2 \mathrm{n}}^{\mathbf{n}}$}

The numerator factors of $C_{2 n}^{n}$ include the natural numbers $(n+1) \sim 2 n$; the denominator factors of $C_{2 n}^{n}$ include the natural numbers $2 \sim n$. The multiplicative factor 1 can be excluded from the denominator, for it does not affect the value of $C_{2 n}^{n}$.

Property 3 Let $p$ be a prime number, $n$ be a positive integer. Suppose $s$ is the maximum integer satisfying $p^{s} \mid n$ !, then $S=\sum_{i=1}^{\infty}\left[\frac{n}{p^{i}}\right]$.

Proof: As $n$ ! includes all the product factors $p^{i}, 2 p^{i}, 3 p^{i}, \ldots$ which are less than or equal to $\mathrm{n}$, so the number of product factors of the multiples of $p^{i}$ is $\left[\frac{n}{p^{i}}\right]$. Suppose $s$ is the maximum integer satisfying $p^{s} \mid n !, s$ is the sum of the exponents of all the multiples of $p$, then $\mathrm{s}=\sum_{i=1}^{\infty}\left[\frac{n}{p^{i}}\right]$.

Property 4 For any prime p, let $s$ be the maximal number satisfying $p^{3} \mid \mathrm{n}$ !, then $\mathrm{n} !=\prod_{p \leq n} \mathrm{p}^{s}$.

Proof: Let $p$ be a prime. Any prime factor in $n$ ! is in $1 \sim n$, thus (n!) $\mid \prod_{p \leq n} \mathrm{p}^{s}$. For any prime $\mathrm{p} \leq \mathrm{n}$, $\mathrm{s}$ is the maximal number satisfying $p^{s} \mid \mathrm{n}$ !, so $\prod_{p \leq n} \mathrm{p}^{s}(\mathrm{n} !)$. Hence $\mathrm{n} !=\prod_{p \leq n} \mathrm{p}^{s}$.

According to property 3 and property 4, we can get

Corollary 4.1 For any prime $\mathrm{p} \leq \mathrm{n}$, let $\mathrm{s}_{\mathrm{p}}$ be the maximal number satisfying $p^{s} v \mid \mathrm{C}_{2 n}^{n}$, then $\mathrm{s}_{\mathrm{p}}=\sum_{i=1}^{\infty}\left(\left[\frac{2 n}{p^{i}}\right]-2\left[\frac{p_{p^{i}}^{n}}{]}\right)\right.$, $\prod_{p s n} p^{s} p \mid C_{2 n}^{n}$.

As we know, $C_{2 n}^{n}=\frac{(2 n)(2 n-1) \ldots(n+1)}{2 \times 3 \ldots \times n}$.

Define composite expression A1 as the expression with all the composite factors in $C_{2 n}^{n}$

$$
\mathrm{A} 1=\frac{(2 n) \times \ldots}{4 \times 6 \times 8 \times 9 \times \ldots}
$$

Define mapping expression A2 as the expression with all the mapping factors in $C_{2 n}^{n}$

$$
\text { A2 }=\frac{(2 n-2) \times(2 n-4) \ldots}{2 \times \ldots}
$$

Define p's multiple expression C1(p) as the expression with all of the p's multiple numbers in $C_{2 n}^{n}$ 


$$
\mathrm{C} 1(\mathrm{p})=\frac{\left(\left[\frac{2 \mathrm{n}}{p}\right] \times \mathrm{p}\right) \times\left(\left[\frac{2 n}{p}\right] \times \mathrm{p}-\mathrm{p}\right) \times \ldots}{p \times 2 p \times \ldots}
$$

Define p's composite expression C2(p) as the expression with all of the p's composite numbers in $C_{2 n}^{n}$

$$
\mathrm{C} 2(\mathrm{p})=\frac{\left(\left[\frac{2 \mathrm{n}}{p}\right] \times \mathrm{p}\right) \times\left(\left[\frac{2 n}{p}\right] \times \mathrm{p}-\mathrm{p}\right) \times \ldots}{2 p \times 3 p \times \ldots}
$$

Define p's mapping expression $\mathrm{C} 3(\mathrm{p})$ as the expression with all of the p's mapping numbers in $C_{2 n}^{n}$

$$
\mathrm{C} 3(\mathrm{p})=\frac{(2 \mathrm{~m}-2 \mathrm{p}) \times(2 \mathrm{~m}-3 \mathrm{p}) \times \ldots}{\left(2 \mathrm{~m}-\left[\frac{2 \pi}{p}\right] \times \mathrm{p}\right) \times\left(2 \mathrm{~m}-\left[\frac{2 n}{p}\right] \times \mathrm{p}+\mathrm{p}\right) \times \ldots}
$$

The orem $4 C_{2 n}^{n}=\frac{(2 n) \times(2 n-1) \times \cdots(n+1)}{2 \times 3 \times \cdots n}$. For an even number $2 n+2$ with the mathematical modeling of table 4 ,

the multiplicative factors with only the factors of complete composite pairs in the numerator and denominator of $C_{2 n}^{n} \geq$ $\frac{4 \frac{n-1}{2}}{n-1}, n \geq 3$.

Proof: (i) When $\mathrm{n}=3$, there is only one complete composite pair $(4,4)$, thus the multiplicative factors with only the factors of complete composite pairs in the numerator and denominator of $C_{2 n}^{n}=4 ; \frac{4 \frac{n-1}{2}}{n-1}=\frac{4 \frac{3-1}{2}}{3-1}=2$. Theorem 4 is right then.

(ii) Consider when $n>3$.The composite factors in $C_{2 n}^{n}$ form an expression

$$
C=\frac{2 n \times \ldots}{4 \times 6 \times 8 \times 9 \times \ldots .}, \mathrm{n}>3
$$

where the composite factors in the denominator include all the composite numbers in $1 \sim \mathrm{n}$, with 4 as the minimal number; the composite factors in the numerator include all the composite numbers in $(n+1) \sim 2 n$, with $2 n$ as the maximal number.

The mapping numbers in $C_{2 n}^{n}$ form an expression

$$
M=\frac{(2 n-2) \ldots}{2 x_{m}}, \mathrm{n}>3
$$

where the mapping numbers in the denominator include all the mapping numbers $1 \sim n$, with 2 as the minimal number; the mapping numbers in the numerator include all the mapping numbers $(\mathrm{n}+1) \sim 2 \mathrm{n}$, with $2 \mathrm{n}-2$ as the maximal mapping number.

There are two situations for $\mathrm{C}$ and $\mathrm{M}$ to form complete composite pairs: (i) if $\mathrm{m}$ is a prime number, the coinciding factors of $\mathrm{C}$ and $\mathrm{M}$ are in the complete composite pairs, and the number of the factors of the complete composite pairs in $(n+1) \sim 2 n$ equals to the number in $1 \sim n$. (ii) If $m$ is a composite number, the factor number of the complete composite pairs in $(\mathrm{n}+1) \sim 2 \mathrm{n}$ has one more factor than in $1 \sim \mathrm{n}$. We can get:

the multiplicative factors with only the factors of complete composite pairs in the numerator and denominator of $C_{2 n}^{n}$ $\geq$ the multiplicative factors with only the factors of the even composite pairs in the numerator and denominator of $C_{2 n}^{n}$ $=\frac{(2 n-2) \times(2 n-4) \times \ldots n n}{4 n}$

(where $n "$ is the minimal even number not less than $n+1$, and $n$ ' is the maximal even number not greater than $\mathrm{n}$. This expression includes all the even numbers $4 \sim(2 n-2)$. )

\section{$\geq \frac{(n-1)(n-2)(n-3) \ldots}{2 \times 3 \times 4 \ldots} \quad(\mathrm{CM}) \quad$ (When $n-1$ is odd, it is " $=$ ";} when $n-1$ is even, it is " $>$ ".)

(CM) includes all the continuous numbers in 2 (n-1). When (n-1) is odd, there is an equal number of factors in the numerator and denominator of (CM). When (n-1) is even, there is one more factor in the numerator of (CM), and it is the minimal number $\frac{n+1}{2}$ which is less than $n "=n+1$. To sum up, there are two situations:

(i) if $n$ is odd, $(\mathrm{CM})=C_{n-1}^{\frac{n-1}{2}}$;

(ii) if $\mathrm{n}$ is even, $(\mathrm{CM})=C_{n-1}^{\frac{n}{2}-1}=C_{n-1}^{\frac{n}{2}}$.

According to corollary 1.2, (CM) $\geq \frac{4 \frac{n-1}{2}}{n-1}$.

Therefore, theorem 4 is proved.

\section{E. Mapping Transformation Numbers}

Let $\mathrm{L}=\left[\frac{2 \mathrm{n}}{p}\right]$, then $2 \mathrm{~m}=\left[\frac{2 \mathrm{n}}{p}\right] \times \mathrm{p}+\mathrm{i}_{\mathrm{p}}=\mathrm{L} \times \mathrm{p}+\mathrm{i}_{\mathrm{p}}$.

A mapping number corresponding to a composite number $\mathrm{k}^{\prime} \mathrm{p}$ is $2 \mathrm{~m}-\mathrm{k}^{\prime} \mathrm{p}=\left(\mathrm{L}-\mathrm{k}^{\prime}\right) \times \mathrm{p}+\mathrm{i}_{\mathrm{p}}=\mathrm{k} \times \mathrm{p}+\mathrm{i}_{\mathrm{p}}$. If we express $\mathrm{a}$ mapping number as $k p+\mathrm{i}_{\mathrm{p}}, \quad 0 \leq \mathrm{k} \leq[2 \mathrm{n} / \mathrm{p}]-2$, define the corresponding mapping transformation number of $\mathbf{k} \mathbf{p}+\mathbf{i}_{\mathbf{p}}$ as $<k p+i_{p}>$ :

$$
<k p+i_{p}>=\left\{\begin{array}{r}
1, k=0 \text { and } 2 \leq i_{p}<p \\
k p, k \neq 0 \text { and } 2 \leq i_{p}<p \\
(k+1) p, p \leq i_{p} \leq p+1
\end{array}\right.
$$

Mapping transformation number (or shortened as mapping transformation) is a characteristic number induced from mapping numbers and convenient for analyzing mapping numbers. For example, as to $\frac{2 \mathrm{~m}-\mathrm{kp}}{2 \mathrm{~m}-\mathrm{k} p \mathrm{p}}=\frac{\left(\mathrm{L}-\mathrm{k} / \mathrm{p}+\mathrm{l}_{\mathrm{p}}\right.}{\left(\mathrm{L}-\mathrm{k}^{\prime}\right) \mathrm{p}+i_{\mathrm{p}}} \leq \frac{(\mathrm{L}-\mathrm{k}) \mathrm{p}}{\left(\mathrm{L}-\mathrm{k}^{\prime}\right) \mathrm{p}}$, the left expression $\frac{2 \mathrm{~m}-\mathrm{kp}}{2 \mathrm{~m}-\mathrm{k} p}$ is in mapping number formation; the right expression $\frac{(\mathrm{L}-\mathrm{k}) \mathrm{p}}{\left(\mathrm{L}-\mathrm{k}^{\prime}\right) \mathrm{p}}$ is in mapping transformation formation. Mapping transformation is a bridge to analyze mapping numbers. Mapping transformation number is not a real factor in multiple exp ression, composite expression, mapping expression or $\boldsymbol{C}_{2 n}^{n}$, but a factor existing in mapping transformation expression.

p's mapping expression $\mathrm{C} 3(\mathrm{p})$ has the following relation:

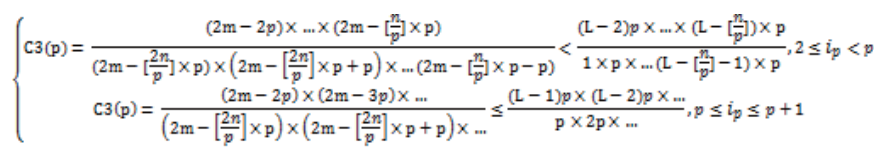

The right expression is the mapping transformation expression C4(p) which satisfies

And

$$
\mathrm{C} 3(\mathrm{p}) \leq \mathrm{C} 4(\mathrm{p})
$$




$$
\mathrm{C} 4(\mathrm{p})=\left\{\begin{array}{l}
\frac{(\mathrm{L}-2) p \times \ldots \times\left(\mathrm{L}-\left[\frac{n}{p}\right]\right) \times \mathrm{p}}{1 \times \mathrm{p} \times \ldots\left(\mathrm{L}-\left[\frac{n}{p}\right]-1\right) \times \mathrm{p}}, 2 \leq i_{p}<p \\
\frac{(\mathrm{L}-1) p \times(\mathrm{L}-2) p \times \ldots}{\mathrm{p} \times 2 \mathrm{p} \times \ldots}, p \leq i_{p} \leq p+1
\end{array}\right.
$$

There is a one-to-one relation among p's composite number, p's mapping number and p's mapping transformation.

\section{(i) Meaning}

p's composite number and p's mapping number are actual numbers in $2 \sim 2 \mathrm{n}$, in the numerator or denominator of $C_{2 n}^{n}$. p's mapping transformation number is a transformed number from corresponding mapping number satisfying $\mathbf{C 3 ( p )} \leq \mathbf{C 4}$ (p).

(ii) Expression form

Table 5 shows the expressions for p's composite number, p's mapping number and p's mapping transformation.

TABLE V. EXPRESSIONS

\begin{tabular}{|c|c|c|}
\hline $\begin{array}{c}\text { p's composite } \\
\text { number }\end{array}$ & p's mapping number & $\begin{array}{c}\text { p's mapping } \\
\text { transformation }\end{array}$ \\
\hline $\mathrm{pk}, 2 \leq \mathrm{k} \leq\left[\frac{2 n}{p}\right]$ & $\mathrm{pk}+\mathrm{i}_{\mathrm{p}}, 0 \leq \mathrm{k} \leq\left[\frac{2 n}{p}\right]-$ & $(\mathrm{PK})$ \\
& $2,2 \leq \mathrm{i}_{\mathrm{p}} \leq \mathrm{p}+1$ & \\
\hline
\end{tabular}

(iii) Difference

The difference between p's adjacent composite numbers is p. The difference between p's adjacent mapping numbers is $\mathrm{p}$. The difference between p's adjacent mapping transformation numbers is $\mathrm{p}$ (except 1 if it is the minimal mapping transformation number when $2 \leq i_{p}<p$ ).

\section{(iv) Head-tail}

The minimal p's composite number is $2 p$; the maximal p's composite number is $\left[\frac{2 n}{p}\right] \times p$. The minimal p's mapping number is $2 \mathrm{~m}-\left[\frac{2 n}{p}\right] \times \mathrm{p}$; the maximal p's mapping number is $2 \mathrm{~m}-2 \mathrm{p}$. The minimal $\mathrm{p}$ 's mapping transformation number is $\mathrm{p}$ $\left(i_{p} \geq p\right)$ or $1\left(i_{p}<p\right)$; the maximal p's mapping transformation number is $(\mathrm{L}-2) \mathrm{p}$.

(v) Numerator $((\mathrm{n}+1) \sim 2 \mathrm{n})$ \& denominator $(2 \sim n)$ of $C_{2 n}^{n}$

Because multiplicative factor 1 can be ignored, the denominator of $C_{2 n}^{n}$ is expressed as 2 n. Generally, suppose one of p's composite number is pk in the numerator or the denominator, its corresponding p's mapping number is $2 \mathrm{~m}$-pk in the denominator or the numerator, and its corresponding $p$ 's mapping transformation number is in the denominator or the numerator. If $\mathrm{p} \mid \mathrm{m}$, m's mapping number and mapping transformation number is itself, and $m$ is still in the numerator, duplicating p's composite number of $m$. If $i_{p}<p$, p's minimal mapping transformation is 1 , and it can be ignored as a multiplicative factor. The position in the numerator or denominator of any p's mapping transformation depends on its corresponding mapping number. That is, the position in the numerator or denominator of a mapping transformation $<k p+i_{p}>$ is the same to its corresponding mapping number $\mathrm{kp}+\mathrm{i}_{\mathrm{p}}$.
F. One to One Matching from p's Composite Numbers to P's Mapping Transformation Numbers

Theorem 5 For an even number $2 n+2 \geq 6$ with the modeling of table 4, $\mathrm{p}$ is a prime less than or equal to $\mathrm{n}$,

$\frac{p^{s} s \text { composite numbers in }(n+1) \sim 2 n}{p^{*} s \text { composite numbers in } 1 \sim n} \times \frac{p^{s} s \text { mapping numbers in } \quad(n+1) \sim 2 n}{p^{s} s \text { mapping numbers in } 1 \sim n}$

$\leq \frac{p^{*} s \text { composite numbers in }(n+1) \sim 2 n}{p^{i} s \text { composite numbers in } 1 \sim n} \times \frac{p^{*} s \text { mapping transformation numbers in }(n+1) \sim 2 n}{p^{*} s \text { mapping transformation numbers in } 1 \sim n}=(\mathrm{J})$.

Let $s_{p}$ be the maximal integer satisfying $p^{s_{p}} \mid(\mathrm{J})$, then $s_{p} \leq\left\{(2 \mathrm{r}-1) \mid \max \left(p^{r}\right) \leq 2 \mathrm{n}\right\}$. " $\max \left(p^{r}\right) \leq 2 \mathrm{n}$ " means $r$ is the maximal value when $p^{r} \leq 2 \mathrm{n}$.

Proof: The theorem is supported by the following table. In this table, $\mathbf{B S}=$ the number of $p$ 's multiples in $(n+1) \sim 2 n-$ the number of $p$ 's multiples in $1 \sim n, \mathbf{H S}=$ the number of $p$ 's composite numbers in $(n+1) \sim 2 n$ - the number of $p$ 's composite numbers in $1 \sim n, \mathbf{Y S}=$ the number of $p$ 's mapping numbers in $(n+1) \sim 2 n$ - the number of $p$ 's mapping numbers in $1 \sim n, \mathbf{Z H}=$ the number of $p$ 's mapping transformation numbers in $(n+1) \sim 2 n-$ the number of $p$ 's mapping transformation numbers in $1 \sim n$. Their corresponding values are shown in table 6.

TABLE VI. VALUESOF BS, HS, YS AND ZH

\begin{tabular}{|c|c|c|c|c|c|}
\hline & & BS & HS & YS & $\overline{\mathrm{ZH}}$ \\
\hline $\mathrm{i}_{\mathrm{p}}=\mathrm{p}+1$ & $\mathrm{p} \nmid \mathrm{m}$ & 0 & 1 & -1 & -1 \\
\hline \multirow[t]{2}{*}{$\mathrm{i}_{\mathrm{p}}=\mathrm{p}$} & $\mathrm{p} \mid \mathrm{m}$ & 1 & 2 & 0 & 0 \\
\hline & $\mathrm{p} \nmid \mathrm{m}$ & 0 & 1 & -1 & -1 \\
\hline \multirow[t]{2}{*}{$2 \leq \mathrm{i}_{\mathrm{p}}<\mathrm{p}$} & \multirow[b]{2}{*}{$\mathrm{p} \nmid \mathrm{m}$} & 1 & 2 & -2 & -1 \\
\hline & & 0 & 1 & -1 & 0 \\
\hline
\end{tabular}

Notice that, " $p \mid m$ " exists only when $i_{p}=p$. Suppose $p \mid m$, then $\mathrm{p}|2 \mathrm{~m}, \mathrm{p}|(2 \mathrm{~m}-\mathrm{p})$, so $\mathrm{i}_{\mathrm{p}}=\mathrm{p}$. When $\mathrm{p} \mid \mathrm{m}, \mathrm{m}$ is considered in $\mathrm{p}$ 's composite expression, in p's mapping expression and p's mapping transformation expression as a member in $(n+1) \sim 2 n$. When $2 \leq i_{p}<p$, the minimal mapping transformation is 1 , then $\mathrm{ZH}=\mathrm{YS}+1$; when $\mathrm{i}_{\mathrm{p}} \geq \mathrm{p}$, ZH=YS.

We give some real cases for the table: for example, $2 \mathrm{~m}=28$ and $\mathrm{p}=3$ for $\mathrm{i}_{\mathrm{p}}=\mathrm{p}+1$ and $\mathrm{p} \nmid \mathrm{m} ; 2 \mathrm{~m}=30$ and $\mathrm{p}=3$ for $\mathrm{i}_{\mathrm{p}}=\mathrm{p}$ and $\mathrm{p} \mid \mathrm{m}$; $2 \mathrm{~m}=10$ and $\mathrm{p}=2$ for $\mathrm{i}_{\mathrm{p}}=\mathrm{p}$ and $\mathrm{p} \nmid \mathrm{m} ; 2 \mathrm{~m}=14$ and $\mathrm{p}=3$ for $2 \leq \mathrm{i}_{\mathrm{p}}<\mathrm{p}$ and $\mathrm{p} \nmid \mathrm{m}$.

\section{As a result, we have}

$\mathrm{HS}+\mathrm{ZH} \leq 1$.

(Notice that in the third row of the table when $\mathrm{p} \mid \mathrm{m}$, HS + $\mathrm{ZH}$ duplicates the "m", their result should be $2-0-1=1 \leq 1$.)

Let $s_{p}$ be the maximal integer satisfying $p^{s_{D}} \mid(\mathrm{J})$, according to corollary 4.1 and property 1 , then $s_{p} \leq\left\{(2 \mathrm{r}-1) \mid \max \left(p^{r}\right) \leq 2 \mathrm{n}\right\}$. " $\max \left(p^{r}\right) \leq 2 \mathrm{n}$ " means $\mathrm{r}$ is the maximal value when $p^{r} \leq 2 \mathrm{n}$.

Thus Theorem 5 is right.

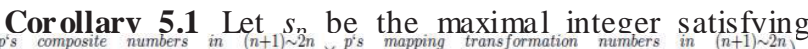

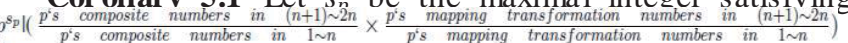

then

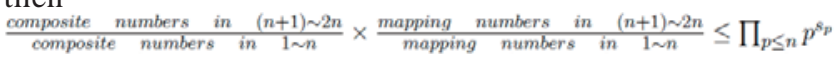

Proof: There is a one-to-one mapping relation between the composite numbers and mapping numbers. 
Let $A$ be the set of all the composite numbers in $2 \sim 2 n$, B be the set of all the mapping numbers in $2 \sim 2 n$. The element number in $\mathrm{A}$ equals to that in $\mathrm{B}$. The elements in A one-to-one correspond to those in $\mathrm{B}$. Let a be any element in $\mathrm{A}$, $\mathrm{a} \in \mathrm{A}$. Its corresponding element in $\mathrm{B}$ is $\mathrm{b}, \mathrm{b} \in \mathrm{B}$, and $\mathrm{b}=2 \mathrm{~m}-\mathrm{a}$.

Consider any prime number $\mathrm{p} \leq \mathrm{n}$. Let $\mathrm{A}(\mathrm{p})$ be the set of all p's composite numbers, B(p) be the set of all p's mapping numbers. The element number in $A(p)$ equals to that in $B(p)$. The elements in $A(p)$ one-to-one correspond to those in $B(p)$. Suppose $a$ is an element in $A(p), a \in A(p)$, and $a=k p$. Its correspondent element in $\mathrm{B}(\mathrm{p})$ is $\mathrm{b}, \mathrm{b} \in \mathrm{B}(\mathrm{p})$, and $\mathrm{b}=2 \mathrm{~m}-\mathrm{a}=2 \mathrm{~m}$ $\mathrm{kp}=(\mathrm{L}-\mathrm{k}) \mathrm{p}+\mathrm{ip}$. The difference between any adjacent elements in $\mathrm{A}(\mathrm{p})$ and $\mathrm{B}(\mathrm{p})$ is $\mathrm{p}$.

Consider the numerator and denominator of $C_{2 n}^{n}$. All the composite numbers in the numerator and denominator are produced by all the prime numbers not greater than $n$. All the mapping numbers in the numerator and denominator are also produced by all the prime numbers not greater than $n$ correspondingly.

Consider p's composite numbers in the numerator and denominator of $C_{2 n}^{n}$. Let ${ }^{\mathrm{t}}$ be the maximal integer satisfying

$$
p^{t_{p}} \mid\left(\frac{p^{c} s \text { composite numbers in }(n+1) \sim 2 n}{p^{i} s \text { composite numbers in } 1 \sim n}\right)
$$

Suppose p's composite numbers in $1 \sim \mathrm{n}$ are $\mathrm{m} 1, \mathrm{~m} 2 \ldots \mathrm{mi}$..., p's composite numbers in $(n+1) \sim 2 n$ are $z 1, z 2 \ldots z i \ldots$. So, in the numerator and denominator of $C_{2 n}^{n}$

$$
p^{t_{p}} \mid\left(\frac{z 1 \times z 2 \times \cdots z i \cdots}{m 1 \times m 2 \times \cdots m i \cdots}\right)
$$

Correspondently, p's mapping numbers in the numerator and denominator of $C_{2 n}^{n}$ are

$$
\frac{(2 m-m 1) \times(2 m-m 2) \times \cdots(2 m-m i) \cdots}{(2 m-z 1) \times(2 m-z 2) \times \cdots(2 m-z i) \cdots}
$$

Suppose p's mapping transformation numbers are M1, $\mathrm{M} 2, \ldots$ to p's mapping numbers $2 \mathrm{~m}-\mathrm{m} 1,2 \mathrm{~m}-\mathrm{m} 2, \ldots$, and $\mathrm{p}$ 's mapping transformation numbers $Z 1, Z 2, \ldots$ to p's mapping numbers $2 \mathrm{~m}-\mathrm{z} 1,2 \mathrm{~m}-\mathrm{z} 2, \ldots$... According to (A)

$$
\frac{(2 m-m 1) \times(2 m-m 2) \times \cdots(2 m-m i) \cdots}{(2 m-z 1) \times(2 m-z 2) \times \cdots(2 m-z i) \cdots} \leq \frac{M 1 \times M 2 \times \cdots M i \cdots}{Z 1 \times Z 2 \times \cdots Z i \cdots}
$$

Let ${ }^{S_{p}}$ be the maximal integer satisfying

$$
p^{s_{p}} \mid\left(\frac{z 1 \times z 2 \times \cdots z i \cdots}{m 1 \times m 2 \times \cdots m i \cdots} \times \frac{M 1 \times M 2 \times \cdots M i \cdots}{Z 1 \times Z 2 \times \cdots Z i \cdots}\right)
$$

Consider all the prime numbers less than or equal to $n$, we have

$$
\prod_{p \leq n} p^{t_{p}}=\left(\frac{\text { composite numbers in }(n+1) \sim 2 n}{\text { composite numbers in } 1 \sim n}\right)
$$

Consider all the prime numbers less than or equal to n, we also have

$\frac{\text { composite numbers in }(n+1) \sim 2 n}{\text { composite numbers in } 1 \sim n} \times \frac{\text { mapping numbers in }(n+1) \sim 2 n}{\text { mapping numbers in } 1 \sim n} \leq \prod_{p \leq n} p^{s_{p}}$

Proved.

\section{PROVING THE GOLDBACH CONJECTURE USING INDUCTION TO ABSURDITY}

The guiding ideology of the proof comes from Theorem 3. The Goldbach conjecture means that there is at least one prime pair among the left-right decomposition pairs for any even number greater than 4 . We are going to prove the conjecture using an induction to absurdity. Under the supposition that any decomposition (a, b) in table 4 is not a prime pair, a contradictory result is induced, so the hypothes is is not right and then the Goldbach conjecture is right.

The following is the detailed proof. For an even number $2 n+2 \geq 6$ with the mathematical modeling of table $4,2 n+2=a+b$, $\mathrm{n} \geq 2,2 \leq \mathrm{a} \leq \mathrm{n}+1, \mathrm{n}+1 \leq \mathrm{b} \leq 2 \mathrm{n}$. $\mathrm{a}$ is in the denominator of $C_{2 n}^{n}$ when $\mathrm{a}<\mathrm{n}+1$. $\mathrm{b}$ is in the numerator of $C_{2 n}^{n}$ when $\mathrm{b}>\mathrm{n}+1$. a and $\mathrm{b}$ are in the numerator of $C_{2 n}^{n}$ when a $=\mathrm{b}=\mathrm{n}+1$. Suppose the even number $2 n+2$ cannot be expressed as the sum of two prime numbers, hence its decomposition pair is either an incomplete composite pair or a complete composite pair, never a prime pair. This means that: any number in $2 \sim 2 n$ is either a composite number or a mapping number produced by a prime number $\mathrm{p} \leq \mathrm{n}$. We can get

$$
C_{2 n}^{n}=\frac{(2 n) \times(2 n-1) \times \cdots(n+1)}{1 \times 2 \times 3 \times \cdots n}
$$

(Two members of a complete composite pair are both composite numbers.)

$=\frac{\text { composite numbers in }(n+1) \sim 2 n}{\text { composite numbers in } 1 \sim n} \times \frac{\text { mapping numbers in } \quad(n+1) \sim 2 n}{\text { mapping numbers in } 1 \sim n}$

$\div$ the multiplicative factors with only the factors of complete composite pairs in the numerator and denominator of $C_{2 n}^{n}$

(According to theorem 4, when $\mathrm{n} \geq 3$ )

$\leq \frac{\text { composite numbers in }(n+1) \sim 2 n}{\text { composite numbers in } 1 \sim n} \times \frac{\text { mapping numbers in }(n+1) \sim 2 n}{\text { mapping numbers in } 1 \sim n} \div \frac{4 \frac{\frac{n-1}{2}}{n-1}}{(B)}$

Let $\mathrm{p}$ be any prime number in $1 \sim n,{ }^{\mathrm{S}} p$ be the maximal integer satisfying

$p^{s_{p}} \mid\left(\frac{p^{\prime} s \text { composite numbers in }(n+1) \sim 2 n}{p^{\prime} s \text { composite numbers in } 1 \sim n} \times \frac{p^{\prime} s \text { mapping transformation numbers in }(n+1) \sim 2 n}{p^{\prime} s \text { mapping transformation numbers in } 1 \sim n}\right)$

Consider all the prime numbers less than or equal to $n$, according to corollary 5.1,

$(B) \leq \prod_{p \leq n} p^{s_{p}} \div \frac{4^{\frac{n-1}{2}}}{n-1}, s_{p} \leq\left\{(2 r-1) \mid \max \left(p^{r}\right) \leq 2 n\right\}$

There are two possible situations for the distribution of prime p:

(i)When $\sqrt{2 n}<p \leq n$, the maximal exponent of p's multiples is 1 . Hence $\mathrm{s}_{p} \leq 1$.

(ii) When $p \leq \sqrt{2 n}, p^{s_{p}}<(2 n)^{2}$.

According to the upper possible values of $\mathrm{S}_{p}$, we get 


$$
\begin{aligned}
& (B) \leq \prod_{p \leq n} p^{s_{p}} \div \frac{4^{\frac{n-1}{2}}}{n-1} \\
& =\prod_{\sqrt{2 n}<p \leq n} p^{s_{p}} \times \prod_{p \leq \sqrt{2 n}} p^{s_{p}} \div \frac{4^{\frac{n-1}{2}}}{n-1} \\
& \leq \prod_{\sqrt{2 n}<p \leq n} p \times \prod_{p \leq \sqrt{2 n}} p^{s_{p}} \div \frac{4^{\frac{n-1}{2}}}{n-1} \\
& =\prod_{p \leq n} p \times \prod_{p \leq \sqrt{2 n}} p^{s_{p}-1} \div \frac{4^{\frac{n-1}{2}}}{n-1}
\end{aligned}
$$

(According to theorem 2)

$$
\begin{aligned}
& <4^{n} \div \frac{4^{\frac{n-1}{2}}}{n-1} \times \prod_{p \leq \sqrt{2 n}} p^{s_{p}-1} \\
& <4^{n} \div \frac{4^{\frac{n-1}{2}}}{n-1} \times \prod_{p \leq \sqrt{2 n}} p^{s_{p}} \\
= & 4^{\frac{n+1}{2}} \times(n-1) \times \prod_{p \leq \sqrt{2 n}} p^{s_{p}}
\end{aligned}
$$

$\left(\frac{\sqrt{2 n}}{2}-1\right.$ is the maximal number of prime numbers $\leq \sqrt{2 n}$ when $\sqrt{2 n} \geq 10$ or $\mathrm{n} \geq 50$ )

$$
\begin{aligned}
& <4^{\frac{n+1}{2}} \times(n-1) \times(2 n)^{\sqrt{2 n}-2} \\
& <4^{\frac{n+1}{2}} \times n \times(2 n)^{\sqrt{2 n}-2} \\
& =4^{\frac{n}{2}} \times(2 n)^{\sqrt{2 n}-1}
\end{aligned}
$$$$
\text { Thus we get } C_{2 n}^{n}<4^{\frac{n}{2}} \times(2 n)^{\sqrt{2 n}-1}, \mathrm{n} \geq 50 \text {. }
$$

Further, according to the lower bound of $C_{2 n}^{n}$ by theorem 1 , we have

$$
\begin{aligned}
& \frac{4^{n}}{2 n} \leq C_{2 n}^{n}<4^{\frac{n}{2}} \times(2 n)^{\sqrt{2 n}-1}, n \geq 50 \\
& \frac{4^{n}}{2 n}<4^{\frac{n}{2}} \times(2 n)^{\sqrt{2 n}-1}, n \geq 50 \\
& 4^{\frac{n}{2}}<(2 n)^{\sqrt{2 n}}, n \geq 50 \\
& \text { Let } \mathrm{x}=\sqrt{2 n}>1 \text {, we get } 2^{\frac{x^{2}}{2}}<x^{2 x} \text {, so } \\
& \frac{x^{2}}{2}<2 x \log _{2} x \\
& x<4 \log _{2} x \\
& x<\log _{2} x^{4} \\
& 2^{x}<x^{4}, x>1
\end{aligned}
$$

When $\mathrm{x} \geq 16$, or when $\mathrm{n} \geq 128$, the inequality " $2^{x}<x^{4}$ ", is not right. At last, an absurdity is induced when $n \geq 128$. Hence, as for even numbers greater than or equal to $2 n+2=258$, the supposition is wrong; therefore, the Goldbach conjecture is right when $\mathrm{n} \geq 128$. The Goldbach conjecture can be verified for the 126 event numbers in 6 256.

Hence, the Goldbach conjecture is proved.

\section{CONCLUSION}

We prove the Goldbach conjecture by an induction to absurdity. Suppose any even number $e=2 n+2$ greater than or equal to 6 does not has a prime pair, but has only incomplete composite pairs or complete composite pairs. All the numbers in $2 \sim 2 n$ can be then expressed by composite numbers or mapping numbers of a prime number less than or equal to $\mathrm{n}$. Under such supposition, an inequality " $2^{x}<x^{4}$ " $(\mathrm{x}=\sqrt{2 n}>1)$ is induced. The inequality " $2^{x}<x^{4}$ "' is not right when $\mathrm{n} \geq 128$. Hence, as for even numbers greater than or equal to $2 n+2=258$, the supposition is not right. The induced benchmark event number 258 is a very small event number.

Contributions of the study include mainly two aspects.

(i) The mathematical modeling of the Goldbach conjecture expressed by table 4 . Any even number greater than four can be expressed as $2 n+2$, and $2 n+2=a+b, n \geq 4,2 \leq a \leq n+1 ; n+1 \leq b \leq 2 n$. a is in the denominator of $C_{2 n}^{n}$ when a<n+1. $\mathrm{b}$ is in the numerator of $C_{2 n}^{n}$ when $\mathrm{b}>\mathrm{n}+1$. a and $\mathrm{b}$ are in the numerator of $C_{2 n}^{n}$ when $\mathrm{a}=\mathrm{b}=\mathrm{n}+1$.

(ii) The proof of the Goldbach conjecture in section 5. Suppose the Goldbach conjecture is wrong. An inequation can be obtained $\frac{4^{n}}{2 n}<4^{\frac{n *}{2}} \times(2 n)^{\sqrt{2 n}-1}, n \geq 50$. This inequation is not true when $n \geq 128$. This means that the supposition "the Goldbach conjecture is wrong" does not stand when $n \geq 128$.

\section{ACKNOWLEDGMENT}

Thanks to the fund - Research on Fast Perception and Track Forecasting of Dynamic Barrier Based on Camera in Unmanned Vehicle, which is supported by Sichuan science and technology Department, No.2017GZ0370.

\section{REFERENCES}

[1] YG Chen, JH Fang, $\mathrm{P}$ Yuan, Y Zheng. "On multiplicative functions with $\mathrm{f}(\mathrm{p}+\mathrm{q}+\mathrm{n} 0)=\mathrm{f}(\mathrm{p})+\mathrm{f}(\mathrm{q})+\mathrm{f}(\mathrm{n} 0)$ ". Joumal of Number Theory 165:270-289, 2016.

[2] Claus, BAUER. "Large sieve inequality with sparse sets of moduli applied to Goldbach conjecture". Frontiers of Mathematics in China, 12(2):1-20, 2017.

[3] https://en.wikipedia.org/wiki/Goldbach\%27s_conjecture . Retrieved on December 13, 2017

[4] Meng ZHANG, "Waring-Goldbach problems for unlike powers with almost equal variables", Frontiers of Mathematics in China, 11(2):449460, 2016

[5] Yu Xinhe. "A new attempt at solving Goldbach conjecture". Journal of Fujian Normal University. 2:1-8, 1993.

[6] H. A. HELFGOTT AND DAVID J. PLATT. "Numerical verification of the ternary Goldbach conjecture up to 8.875.10\%"\%. Experimental Mathematics. 22(4):406-409, Dec. 2013.

[7] Kumchev Angel V., Wooley Trevor D., "On the Waring-Goldbach problem for eighth and higher powers". Journal of the London mathematical society.93(3):811-824, 2016.

[8] Yu Wang, “A compact distribution law of prime numbers". Journal of Chendu University of Thechnology. 3:373-376, 2017 